Www.jmscr.igmpublication.org

Impact Factor 5.84

Index Copernicus Value: 71.58

ISSN (e)-2347-176x ISSN (p) 2455-0450

crossref DOI: https://dx.doi.org/10.18535/jmscr/v5i10.18

Journal Of Medical Science And Clinical Research

\title{
Fibrous Synovial Sarcoma Omentum Presenting As a Large Intra- Abdominal Tumor
}

\author{
Authors \\ AK Kapoor, Akanksha Singh, Rajesh Kumar Srivastava \\ RML Mehrotra Pathology Pvt. Ltd., Nirala Nagar, Lucknow, India \\ Corresponding Author \\ Ashok Kumar Kapoor \\ RML Mehrotra Pathology Pvt Limited, B 171, Nirala Nagar, Lucknow, India \\ Email:drashokkapoor2016@gmail.com
}

\begin{abstract}
A case with large intra-abdominal monophasic synovial sarcoma has been described. Ultrasonography revealed a large tumor having both solid and cystic areas. Exploratory laparotomy revealed that the tumor was arising from the omentum. Microscopic examination showed proliferation of spindle cells. Immunohistochemical (IHC) examination showed strong positivity for Vimentin, CD99 and Bcl-2. Focal positivity for cytokeratin was also seen. Result of FISH indicated break at SYT region. Findings were suggestive of a monophasic fibrous synovial sarcoma.

Keywords: Monophasic mesenchymal malignant neoplasm.
\end{abstract}

\section{Introduction}

Synovial sarcoma (SS) commonly arises from para-articular areas of extremities. Microscopically, it may be of biphasic or monophasic types. Biphasic SS has both mesenchymal and epithelial components ${ }^{1}$. Translocation involving chromosome $\mathrm{x}$ and $18[\mathrm{t}(\mathrm{x}: 18)$ (p11.2; q11.2) is found in most of synovial sarcomas. This translocation results in the formation of a hybrid protein SYT-SSX ${ }^{2}$. Here, we describe a case of omental synovial sarcoma.

\section{Case Report}

A 50 year old lady had a dragging sensation in abdomen and vague abdominal pain since 3 months. Ultrasonography revealed a large abdominal tumor having both solid and cystic areas. Tumor extended from pelvis to epigastrium. CT abdomen with contrast showed a large tumor, measuring $32.4 \times 29.7 \times 19.6 \mathrm{~cm}$. Exploratory laparotomy revealed that the tumor was arising from the omentum. Grossly, multiple grayish white soft tissue pieces were received. Cut surface showed grayish white solid and cystic areas.

Microscopic examination showed tumor tissue, consisting of interlacing bundles of proliferated spindle cells. Mitoses were also seen (figure 1a and $b$ ). Tumor showed areas of hemorrhage and coagulative necrosis. IHC examination showed mild focal positive (+) staining with anti-pan cytokeratin antibody (figure c). Strong positivity for Vimentin, CD99 and Bcl-2 antigens was also seen (figure $1 \mathrm{~d}$, e and $\mathrm{f}$ ). Tumor cells were 
negative for desmin, myogenin, smooth muscle actin, chymotrypsin, EMA and S-100 antigens. Kreatech ${ }^{\mathrm{TM}} \mathrm{SS} 18(18 \mathrm{q} 11)$ break FISH probe was purchased through Leica customer service. Probe was optimized to detect translocation of SS18
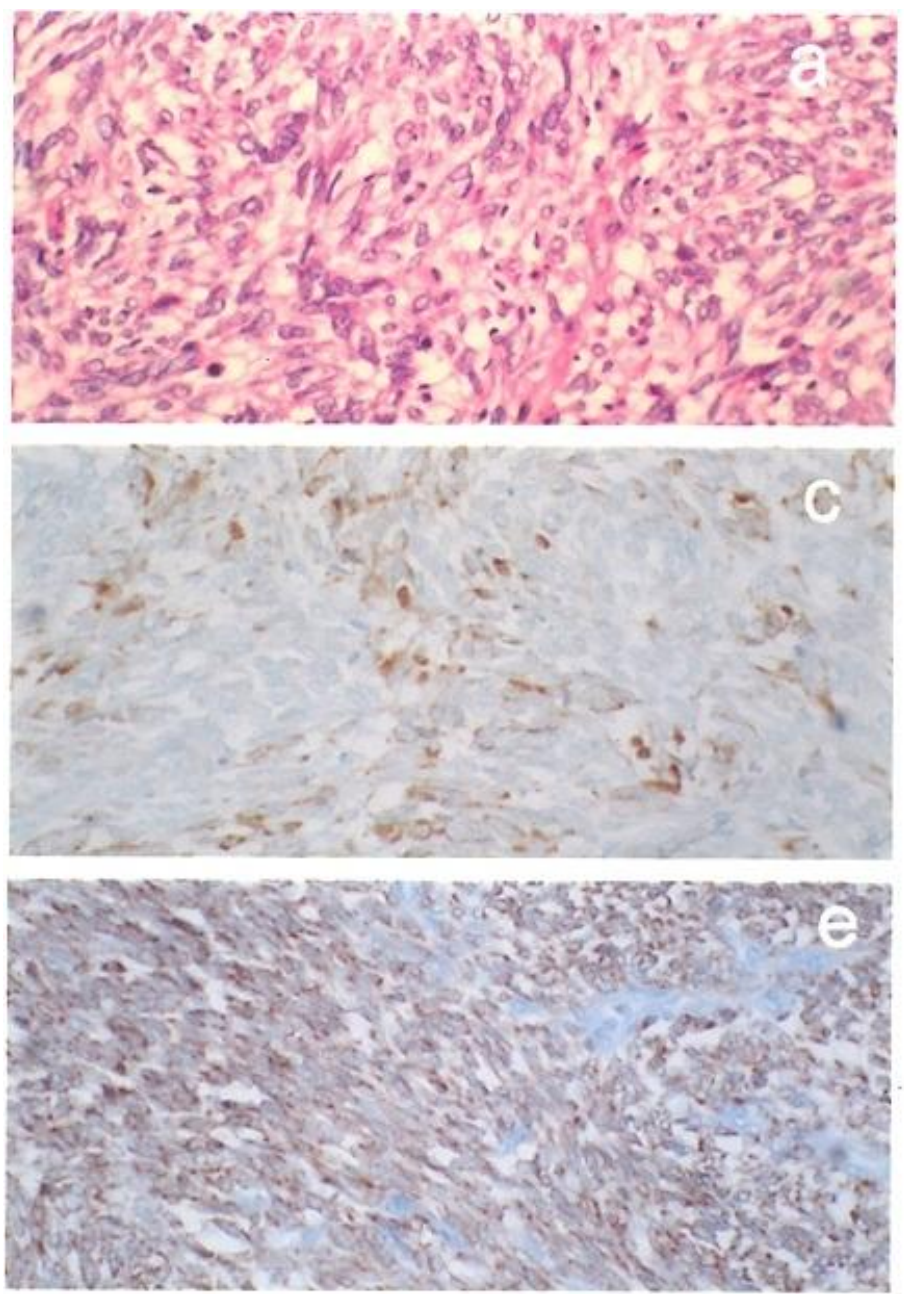

gene region in a dual color split assay on tissue sections. A break is defined when yellow signal breaks into separate red and green signals. Result of FISH suggested break at SYT region.
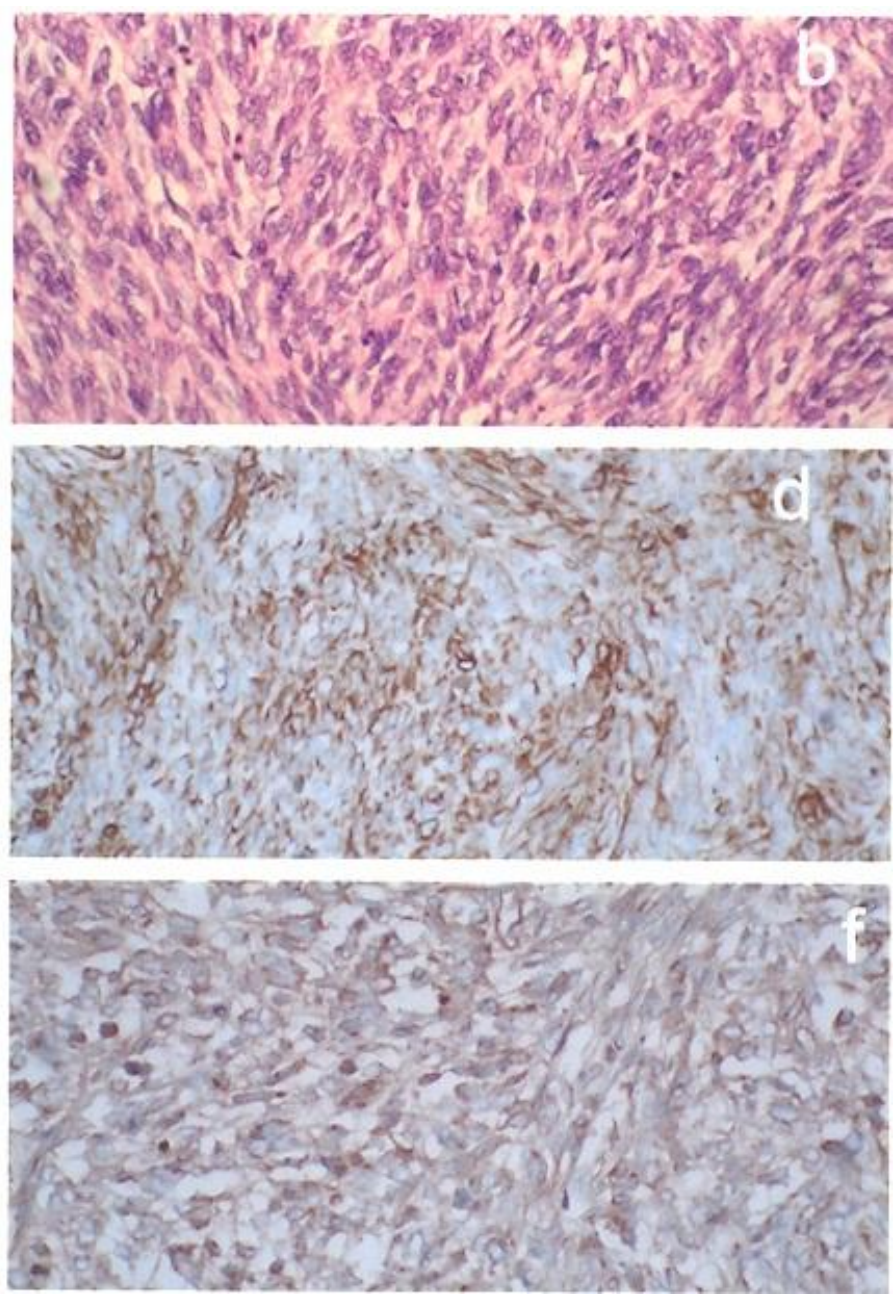

Figure I (a) \& (b): Photomicrograph showing tumor $(\mathrm{HE} \times 450)$; (c) shows focal positivity (+) for cytokeratin, (d) shows strong positive reaction with vimentin antigen; (e) and (f) showed positivity for CD99 and Bcl-2 antigens on tumor cells (magnification $\times 450$ ).

\section{Discussion}

Absence of desmin, myogenin, SMA, chymotrypsin excluded the diagnosis of other relatively more common soft tissue neoplasms. Bcl-2 blocks apoptosis and is found in lymphoma and leukemia. Bcl-2 antigen positivity favored its mesenchymal origin. Vimentin positivity also suggested mesenchymal origin of this tumor. Focal Pan cytokeratin antigen positivity (+) of tumor cells suggested the epithelial origin of small number of tumor cells.
In the present study, the site of origin of the tumor was omentum. Only 3 cases have been described earlier. Vimentin and Bcl-2 positivity along with negative reaction with desmin, actin and S-100 suggested the antigenic similarity of the current case with an earlier study from Taiwan ${ }^{3}$. Cytokeratin positivity has also been reported earlier from Romania ${ }^{4}$. In the later study, the tumor was adherent to omentum. Another case of omental synovial sarcoma has been reported from Kolkata, India. Tumor from previous patient gave 
strong reactivity with anti bcl-2 and anti-SMA antibodies ${ }^{5}$.

Focal cytokeratin positivity was also found in 2 earlier studies ${ }^{3,4}$ while another study was cytokeratin negative ${ }^{5}$. Bcl-2 positivity was also seen in earlier studies ${ }^{3,5}$. In the current study, CD99 antigen was detected while this antigen was not expressed in the omentaltumor of another study $^{5}$.

A vaccine of SYT-SSX junction peptide has been tried $^{6}$. In addition, retinoic acid and its analogues could induce differentiation of SS cell lines and inhibit the growth of tumor ${ }^{7}$.

\section{Conclusion}

Synovial sarcoma is a high grade malignant neoplasm. Omental synovial sarcoma can be treated by surgery. However, in case of recurrence retinoic acid analogues may be tried.

\section{Financial support and sponsorship: Nil}

Conflicts of interest: There are no conflicts of interest.

\section{References}

1. Goldblum JR, Folpe AL, Weiss SW. In Enzinger and Weiss's soft tissue tumors. Chapter 33, 2014, $6^{\text {th }}$ ed, p1028-1112.

2. Schiller AL, Teitelbaum SL. In 'Bones and joints` chapter 26, Pathology, Rubin E, Farber J (eds), $3^{\text {rd }}$ edition, Lippincott Raven, Philadelphia (publisher) 1999 p1336-1413.

3. Wang Yzu Jan, Wen Shi-Chi, Chien Shang-Tao,Sheu Jin-Wen, Hsuea ChaoWen, Feng Nan-Hsiung. Primary intraabdominal synovial sarcoma. J Clin Med Ass 2006 (69) 492-495.

4. Buiga-Potcoava R, Crisan D, Olinici D. Primary intraabdominal synovial sarcoma : a case report. Romanian $\mathrm{J}$ of Gastroenterology, 2005 (14) 67-69.

5. DivyIG: Synovial sarcoma of the omentum : a rare entity 2015 (52) 166-167.
6. Kawaguchi S, Wada T, Ida Y, Nagoya S, Trukahara T, Kimura S, Sahara H, Shimozava K, Asanuma H. Phase I vaccination trial of SYT-SSX junction peptide in patients with disseminated synovial sarcoma. J Transl Med 2005; 3:1-9.

7. Ishibe $T$, Nakayama $T$, Aoyama $T$, Nakamura T, Toguchida J. Neuronal differentiation of synovial sarcoma and its therapeutic application : A clinicopathological study. Histopathology 2004; 45:245-253. 